Kragujevac Journal of Mathematics

Volume 45(5) (2021), Pages 739-749.

\title{
CONVERGENCE ESTIMATES FOR GUPTA-SRIVASTAVA OPERATORS
}

\author{
DANYAL SOYBAŞ ${ }^{1}$ AND NEHA MALIK ${ }^{2}$
}

Dedicated to Prof. Vijay Gupta

\begin{abstract}
The Grüss-Voronovskaya-type approximation results for the modified Gupta-Srivastava operators are considered. Moreover, the magnitude of differences of two linear positive operators defined on an unbounded interval has been estimated. Quantitative type results are established as we initially obtain the moments of generalized discrete operators and then estimate the difference of these operators with the Gupta-Srivastava operators.
\end{abstract}

\section{INTRODUCTION}

For $f \in C[0, \infty), n \in \mathbb{N}, c \in \mathbb{N} \cup\{0\} \cup\{-1\}$ and $l$ an integer, the generalized form of the discrete operators are given by $($ cf. $[5,15])$ :

$$
M_{n, l, c}(f, x)=\sum_{k=0}^{\infty} p_{n+l c, k}(x, c) f\left(\frac{k}{n}\right)
$$

where $p_{n+l c, k}(x, c)=\frac{\left(\frac{n}{c}+l\right)_{k}}{k !} \cdot \frac{(c x)^{k}}{(1+c x)^{\frac{n}{c}+l+k}}$, the rising factorial given by

$$
(\gamma)_{k}=\gamma(\gamma+1)(\gamma+2) \cdots(\gamma+k-1), \quad(\gamma)_{0}=1
$$

These operators (1.1) reproduce only the constant function unlike other exponential functions. In case $l=0$, we immediately get Szász-Mirakyan operators for $l=0$, $c=0$; classical Baskakov operators for $l=0, c=1$, and Bernstein polynomials for $l=0, c=-1$. In these special cases, these operators reproduce linear function too.

Key words and phrases. Gupta-Srivastava operators, Grüss-Voronovskaya, difference of operators. 2010 Mathematics Subject Classification. Primary: 41A36. Secondary: 41A30.

DOI 10.46793/KgJMat2105.739S

Received: February 12, 2019.

Accepted: May 12, 2019. 
A family of linear positive operators for locally integrable functions was defined in the year 2003 [18]. Durrmeyer variants of many hybrid operators have been extensively studied in literature since then (cf. $[6,9,14,17]$ ). Varied approximation properties of these operators have been studied and investigated (cf. [1,2,4,8,12,13,16,19,20], etc.). For $c$, an integer and $x \in[0, \infty)$, V. Gupta and H. M. Srivastava [10] introduced a modification of these family of operators as:

$$
\begin{aligned}
R_{n, l, c}(f, x)= & {[n+(l+1) c] \sum_{k=1}^{\infty} p_{n+l c, k}(x, c) \int_{0}^{\infty} p_{n+(l+2) c, k-1}(t, c) f(t) d t } \\
& +p_{n+l c, 0}(x, c) f(0),
\end{aligned}
$$

where $p_{n+l c, k}(x, c)$ is as defined previously above. For $c=0$, we get the Phillips operators preserving linear functions and for $c=1$, we immediately obtain the BaskakovDurrmeyer type operators. For $l=0$, the operators (1.2) reduce to the operators defined in [8, Example 2]. Very recently, Gupta [7] established a general estimate for the difference of linear positive operators as follows.

Theorem A. ([7]). Let $f^{(s)} \in C_{B}[0, \infty), s \in\{0,1,2\}$ (the class of bounded continuous functions defined on the interval $[0, \infty))$ and $x \in[0, \infty)$, then for $n \in \mathbb{N}$, we have

$$
\left|\left(G_{n}-V_{n}\right)(f, x)\right| \leq\left\|f^{\prime \prime}\right\| \alpha(x)+\omega\left(f^{\prime \prime}, \delta_{1}\right)(1+\alpha(x))+2 \omega\left(f, \delta_{2}(x)\right),
$$

where $\|\cdot\|=\sup _{x \in[0, \infty)}|f(x)|<\infty, \alpha(x)=\frac{1}{2} \sum_{k=0}^{\infty} p_{n, k}(x, c)\left(\mu_{2}^{G_{n, k}}+\mu_{2}^{H_{n, k}}\right)$ and

$$
\delta_{1}^{2}=\frac{1}{2} \sum_{k=0}^{\infty} p_{n, k}(x, c)\left(\mu_{4}^{G_{n, k}}+\mu_{4}^{H_{n, k}}\right), \quad \delta_{2}^{2}=\sum_{k=0}^{\infty} p_{n, k}(x, c)\left(b^{G_{n, k}}-b^{H_{n, k}}\right)^{2} .
$$

We consider a family of functions $G_{n, k}: D \rightarrow \mathbb{R}$, ( $k$ being a non-negative integer), which are positive linear functionals defined on a subspace $D$ of $C[0, \infty)$, which contains polynomials upto degree 6 and $C_{2}[0, \infty)$, such that, $G_{n, k}\left(e_{0}\right)=1, b^{G_{n, k}}:=$ $G_{n, k}\left(e_{1}\right), \mu_{r}^{G_{n, k}}:=G_{n, k}\left(e_{1}-b^{G_{n, k}} e_{0}\right)^{r}, r \in \mathbb{N}$. Also, let $H_{n, k}$ be a similar family of functions.

We extend the studies of [7] as we study a quantitative Voronovskaya type theorem in terms of weighted modulus of continuity and estimate the difference of the two operators having the same basis function, viz. the generalized Baskakov operators and the genuine Gupta-Srivastava operators.

\section{Moments}

In this section, we give the moments of generalized operators (1.1) with the help of a recurrence formula.

Lemma 2.1. For $m \in \mathbb{N}$, the operators (1.1) satisfy the following recurrence relation:

$$
M_{n, l, c}\left(e_{m+1}, x\right)=\frac{x(1+c x)}{n} M_{n, l, c}^{\prime}\left(e_{m}, x\right)+\left(1+\frac{l c}{n}\right) x M_{n, l, c}\left(e_{m}, x\right),
$$


where $e_{m}(y)=y^{m}$.

Proof. On taking the derivative of the operators $M_{n, l, c}$, we get

$$
M_{n, l, c}^{\prime}(f, x)=\sum_{k=0}^{\infty} p_{n+l c, k}^{\prime}(x, c) f\left(\frac{k}{n}\right)^{m},
$$

which implies that

$$
x(1+c x) M_{n, l, c}^{\prime}(f, x)=\sum_{k=0}^{\infty} x(1+c x) p_{n+l c, k}^{\prime}(x, c) f\left(\frac{k}{n}\right)^{m} .
$$

Now, using the identity $x(1+c x) p_{n+l c, k}^{\prime}(x, c)=[k-(n+l c) x] p_{n+l c, k}(x, c)$, we obtain

$$
\begin{aligned}
x(1+c x) M_{n, l, c}^{\prime}(f, x) & =\sum_{k=0}^{\infty}[k-(n+l c) x] p_{n+l c, k}(x, c) f\left(\frac{k}{n}\right)^{m} \\
& =n \sum_{k=0}^{\infty} p_{n+l c, k}(x, c)\left(\frac{k}{n}\right)^{m+1}-(n+l c) x p_{n+l c, k}(x, c)\left(\frac{k}{n}\right)^{m} \\
& =n M_{n, l, c}\left(e_{m+1}, x\right)-(n-l c) x M_{n, l, c}\left(e_{m}, x\right),
\end{aligned}
$$

which derives the recurrence relation.

Remark 2.1. Using Lemma 2.1, first few moments of the operators (1.1) are given by

$$
\begin{aligned}
& M_{n, l, c}\left(e_{0}, x\right)=1, \\
& M_{n, l, c}\left(e_{1}, x\right)= x\left(1+\frac{l c}{n}\right), \\
& M_{n, l, c}\left(e_{2}, x\right)= x^{2}\left(1+\frac{c^{2} l}{n^{2}}+\frac{c^{2} l^{2}}{n^{2}}+\frac{c}{n}+\frac{2 c l}{n}\right)+x\left(\frac{c l}{n^{2}}+\frac{1}{n}\right), \\
& M_{n, l, c}\left(e_{3}, x\right)= x^{3}\left(1+\frac{2 c^{3} l}{n^{3}}+\frac{3 c^{3} l^{2}}{n^{3}}+\frac{c^{3} l^{3}}{n^{3}}+\frac{2 c^{2}}{n^{2}}+\frac{6 c^{2} l}{n^{2}}+\frac{3 c^{2} l^{2}}{n^{2}}+\frac{3 c}{n}+\frac{3 c l}{n}\right) \\
&+x^{2}\left(\frac{3 c^{2} l}{n^{3}}+\frac{3 c^{2} l^{2}}{n^{3}}+\frac{3 c}{n^{2}}+\frac{6 c l}{n^{2}}+\frac{3}{n}\right)+x\left(\frac{c l}{n^{3}}+\frac{1}{n^{2}}\right), \\
&\left.+\frac{11 c^{2}}{n^{2}}+\frac{18 c^{2} l}{n^{2}}+\frac{6 c^{2} l^{2}}{n^{2}}+\frac{6 c}{n}+\frac{4 c l}{n}\right) \\
&+x^{3}\left(\frac{12 c^{3} l}{n^{4}}+\frac{18 c^{3} l^{2}}{n^{4}}+\frac{6 c^{3} l^{3}}{n^{4}}+\frac{12 c^{2}}{n^{3}}+\frac{36 c^{2} l}{n^{3}}+\frac{18 c^{2} l^{2}}{n^{3}}+\frac{18 c}{n^{4}}+\frac{18 c l}{n^{2}}\right. \\
&\left.\left.+\frac{6}{n}\right)+e^{4}, x\right)+\frac{22 c^{3} l}{n^{3}}+\frac{18 c^{3} l^{2}}{n^{3}}+\frac{4 c^{3} l^{3}}{n^{3}} \\
& x^{4} l \\
& n^{4} \\
& M_{n, l, c}\left(e_{5}, x\right)= x^{5}\left(1+\frac{7 c^{2} l^{2}}{n^{4}}+\frac{7 c}{n^{3}}+\frac{14 c l}{n^{3}}+\frac{7}{n^{2}}\right)+x\left(\frac{50 c^{5} l^{2}}{n^{5}}+\frac{35 c^{5} l^{3}}{n^{5}}+\frac{10 c^{5} l^{4}}{n^{5}}+\frac{c^{5} l^{5}}{n^{5}}+\frac{24 c^{4}}{n^{4}}+\frac{100 c^{4} l}{n^{4}}\right.
\end{aligned}
$$




$$
\begin{aligned}
& +\frac{105 c^{4} l^{2}}{n^{4}}+\frac{40 c^{4} l^{3}}{n^{4}}+\frac{5 c^{4} l^{4}}{n^{4}}+\frac{50 c^{3}}{n^{3}}+\frac{105 c^{3} l}{n^{3}}+\frac{60 c^{3} l^{2}}{n^{3}}+\frac{10 c^{3} l^{3}}{n^{3}} \\
& \left.+\frac{35 c^{2}}{n^{2}}+\frac{40 c^{2} l}{n^{2}}+\frac{10 c^{2} l^{2}}{n^{2}}+\frac{10 c}{n}+\frac{5 c l}{n}\right) \\
& +x^{4}\left(\frac{60 c^{4} l}{n^{5}}+\frac{110 c^{4} l^{2}}{n^{5}}+\frac{60 c^{4} l^{3}}{n^{5}}+\frac{10 c^{4} l^{4}}{n^{5}}+\frac{60 c^{3}}{n^{4}}+\frac{220 c^{3} l}{n^{4}}+\frac{180 c^{3} l^{2}}{n^{4}}\right. \\
& \left.+\frac{40 c^{3} l^{3}}{n^{4}}+\frac{110 c^{2}}{n^{3}}+\frac{180 c^{2} l}{n^{3}}+\frac{60 c^{2} l^{2}}{n^{3}}+\frac{60 c}{n^{2}}+\frac{40 c l}{n^{2}}+\frac{10}{n}\right) \\
& +x^{3}\left(\frac{50 c^{3} l}{n^{5}}+\frac{75 c^{3} l^{2}}{n^{5}}+\frac{25 c^{3} l^{3}}{n^{5}}+\frac{50 c^{2}}{n^{4}}+\frac{150 c^{2} l}{n^{4}}+\frac{75 c^{2} l^{2}}{n^{4}}+\frac{75 c}{n^{3}}\right. \\
& \left.+\frac{75 c l}{n^{3}}+\frac{25}{n^{2}}\right) \\
& +x^{2}\left(\frac{15 c^{2} l}{n^{5}}+\frac{15 c^{2} l^{2}}{n^{5}}+\frac{15 c}{n^{4}}+\frac{30 c l}{n^{4}}+\frac{15}{n^{3}}\right)+x\left(\frac{c l}{n^{5}}+\frac{1}{n^{4}}\right), \\
& M_{n, l, c}\left(e_{6}, x\right)=x^{6}\left(1+\frac{120 c^{6} l}{n^{6}}+\frac{274 c^{6} l^{2}}{n^{6}}+\frac{225 c^{6} l^{3}}{n^{6}}+\frac{85 c^{6} l^{4}}{n^{6}}+\frac{15 c^{6} l^{5}}{n^{6}}+\frac{c^{6} l^{6}}{n^{6}}+\frac{120 c^{5}}{n^{5}}\right. \\
& +\frac{548 c^{5} l}{n^{5}}+\frac{675 c^{5} l^{2}}{n^{5}}+\frac{340 c^{5} l^{3}}{n^{5}}+\frac{75 c^{5} l^{4}}{n^{5}}+\frac{6 c^{5} l^{5}}{n^{5}}+\frac{274 c^{4}}{n^{4}}+\frac{675 c^{4} l}{n^{4}} \\
& +\frac{510 c^{4} l^{2}}{n^{4}}+\frac{150 c^{4} l^{3}}{n^{4}}+\frac{15 c^{4} l^{4}}{n^{4}}+\frac{225 c^{3}}{n^{3}}+\frac{340 c^{3} l}{n^{3}}+\frac{150 c^{3} l^{2}}{n^{3}}+\frac{20 c^{3} l^{3}}{n^{3}} \\
& \left.+\frac{85 c^{2}}{n^{2}}+\frac{75 c^{2} l}{n^{2}}+\frac{15 c^{2} l^{2}}{n^{2}}+\frac{15 c}{n}+\frac{6 c l}{n}\right) \\
& +x^{5}\left(\frac{360 c^{5} l}{n^{6}}+\frac{750 c^{5} l^{2}}{n^{6}}+\frac{525 c^{5} l^{3}}{n^{6}}+\frac{150 c^{5} l^{4}}{n^{6}}+\frac{15 c^{5} l^{5}}{n^{6}}+\frac{360 c^{4}}{n^{5}}\right. \\
& +\frac{1500 c^{4} l}{n^{5}}+\frac{1575 c^{4} l^{2}}{n^{5}}+\frac{600 c^{4} l^{3}}{n^{5}}+\frac{75 c^{4} l^{4}}{n^{5}}+\frac{750 c^{3}}{n^{4}}+\frac{1575 c^{3} l}{n^{4}} \\
& +\frac{900 c^{3} l^{2}}{n^{4}}+\frac{150 c^{3} l^{3}}{n^{4}}+\frac{525 c^{2}}{n^{3}}+\frac{600 c^{2} l}{n^{3}}+\frac{150 c^{2} l^{2}}{n^{3}}+\frac{150 c}{n^{2}}+\frac{75 c l}{n^{2}} \\
& \left.+\frac{15}{n}\right)+x^{4}\left(\frac{390 c^{4} l}{n^{6}}+\frac{715 c^{4} l^{2}}{n^{6}}+\frac{390 c^{4} l^{3}}{n^{6}}+\frac{65 c^{4} l^{4}}{n^{6}}+\frac{390 c^{3}}{n^{5}}+\frac{1430 c^{3} l}{n^{5}}\right. \\
& +\frac{1170 c^{3} l^{2}}{n^{5}}+\frac{260 c^{3} l^{3}}{n^{5}}+\frac{715 c^{2}}{n^{4}}+\frac{1170 c^{2} l}{n^{4}}+\frac{390 c^{2} l^{2}}{n^{4}}+\frac{390 c}{n^{3}}+\frac{260 c l}{n^{3}} \\
& \left.+\frac{65}{n^{2}}\right)+x^{3}\left(\frac{180 c^{3} l}{n^{6}}+\frac{270 c^{3} l^{2}}{n^{6}}+\frac{90 c^{3} l^{3}}{n^{6}}+\frac{180 c^{2}}{n^{5}}+\frac{540 c^{2} l}{n^{5}}+\frac{270 c^{2} l^{2}}{n^{5}}\right. \\
& \left.+\frac{270 c}{n^{4}}+\frac{270 c l}{n^{4}}+\frac{90}{n^{3}}\right)+x^{2}\left(\frac{31 c^{2} l}{n^{6}}+\frac{31 c^{2} l^{2}}{n^{6}}+\frac{31 c}{n^{5}}+\frac{62 c l}{n^{5}}+\frac{31}{n^{4}}\right)
\end{aligned}
$$




$$
+x\left(\frac{c l}{n^{6}}+\frac{1}{n^{5}}\right) .
$$

Remark 2.2. Denote $\mu_{n, m}^{l, c}(x):=R_{n, l, c}\left((t-x)^{m}, x\right)$. Then, using [10, (6)], the central moments are given by $\mu_{n, 0}^{l, c}(x)=1, \mu_{n, 1}^{l, c}(x)=0$, and $\mu_{n, 2}^{l, c}(x)=\frac{2 x(1+c x)}{n+(m-1) c}$. Higher central moments can be obtained easily.

\section{Grüss-Voronovskaya-Type Approximation Results}

The Voronovskaya theorem in quantitative form for a class of sequences of linear positive operators is one of the most significant pointwise results. We obtain these by making using of Taylor series expansion. Let us see at some notations.

Let $C[0, \infty)$ be the set of all continuous functions $f$ defined on $[0, \infty)$ and $B_{2}[0, \infty):=\left\{f:|f(x)| \leq M_{f}\left(1+x^{2}\right)\right.$ with $\left.M_{f}>0\right\}$. Also, let $C_{2}[0, \infty)$ denote the subspace of all continuous functions in $B_{2}[0, \infty)$. Further $C_{2}^{*}[0, \infty)$ denotes the closed subspace of $C_{2}[0, \infty)$ for which $\lim _{x \rightarrow \infty}|f(x)|\left(1+x^{2}\right)^{-1}<C$ for some constant $C$ and $\|\cdot\|_{2}=\sup _{x \in[0, \infty)}|f(x)|\left(1+x^{2}\right)^{-1}$. In [11], Ispir considered for each $f \in C_{2}[0, \infty)$, the following weighted modulus of continuity:

$$
\Omega(f, \delta)=\sup _{x \geqslant 0,|h|<\delta} \frac{|f(x+h)-f(x)|}{\left(1+x^{2}\right)\left(1+h^{2}\right)} .
$$

The quantitative Voronovskaya-type theorem in weighted spaces is as follows.

Theorem 3.1. If $f \in C[0, \infty)$ and $f^{\prime \prime} \in C_{2}^{*}[0, \infty)$, then, for $x \in[0, \infty)$, we have

$$
\begin{aligned}
\left|R_{n, l, c}(f, x)-f(x)-\frac{x(1+c x)}{[n+(m-1) c]} f^{\prime \prime}(x)\right| \leq & 16\left(1+x^{2}\right) \Omega\left(f^{\prime \prime},\left(\frac{\mu_{n, 6}^{l, c}(x)}{\mu_{n, 2}^{l, c}(x)}\right)^{1 / 4}\right) \\
& \times \mu_{n, 2}^{l, c}(x) .
\end{aligned}
$$

Proof. Using the Taylor series expansion of $f$, we can write

$$
f(t)=f(x)+(t-x) f^{\prime}(x)+(t-x)^{2} \frac{f^{\prime \prime}(x)}{2 !}+H(t, x),
$$

where $H(t, x)=\frac{(t-x)^{2}}{2 !}\left(f^{\prime \prime}(\xi)-f^{\prime \prime}(x)\right), \xi$ is a number lying between $t$ and $x$.

Applying the operators $R_{n, l, c}$ to the above expansion, we have

$$
R_{n, l, c}(f, x)-f(x)-f^{\prime}(x) \mu_{n, 1}^{l, c}(x)+\frac{f^{\prime \prime}(x)}{2 !} \mu_{n, 2}^{l, c}(x)=R_{n, l, c}(H(t, x), x) .
$$

Using Remark 2.2, we obtain

$$
\left|R_{n, l, c}(f, x)-f(x)+\frac{f^{\prime \prime}(x)}{2 !} \mu_{n, 2}^{l, c}(x)\right| \leq R_{n, l, c}(|H(t, x)|, x) .
$$


Now, using the property of weighted modulus of continuity given in [11], it follows that

$$
\begin{aligned}
\left|\frac{f^{\prime \prime}(\xi)-f^{\prime \prime}(x)}{2 !}\right| & \leq \frac{1}{2} \Omega\left(f^{\prime \prime},|\xi-x|\right)\left(1+(\xi-x)^{2}\right)\left(1+x^{2}\right) \\
& \leq \frac{1}{2} \Omega\left(f^{\prime \prime},|t-x|\right)\left(1+(t-x)^{2}\right)\left(1+x^{2}\right) \\
& \leq\left(1+\frac{|t-x|}{\delta}\right)\left(1+\delta^{2}\right) \Omega\left(f^{\prime \prime}, \delta\right)\left(1+(t-x)^{2}\right)\left(1+x^{2}\right)
\end{aligned}
$$

Moreover,

$$
\left|\frac{f^{\prime \prime}(\xi)-f^{\prime \prime}(x)}{2 !}\right| \leq \begin{cases}2\left(1+\delta^{2}\right)\left(1+x^{2}\right) \Omega\left(f^{\prime \prime}, \delta\right), & |t-x|<\delta \\ 2\left(1+\delta^{2}\right)\left(1+x^{2}\right) \frac{(t-x)^{4}}{\delta^{4}} \Omega\left(f^{\prime \prime}, \delta\right), & |t-x| \geqslant \delta\end{cases}
$$

For $0<\delta<1$, we get

$$
\left|\frac{f^{\prime \prime}(\xi)-f^{\prime \prime}(x)}{2 !}\right| \leq 8\left(1+x^{2}\right)\left(1+\frac{(t-x)^{4}}{\delta^{4}}\right) \Omega\left(f^{\prime \prime}, \delta\right) .
$$

So, we have

$$
|H(t, x)| \leq 8\left(1+x^{2}\right)\left((t-x)^{2}+\frac{(t-x)^{6}}{\delta^{4}}\right) \Omega\left(f^{\prime \prime}, \delta\right) .
$$

Thus, (3.1) implies

$$
\begin{aligned}
& \left|R_{n, l, c}(f, x)-f(x)+\frac{f^{\prime \prime}(x)}{2 !}\left(\frac{2 x(1+c x)}{n+(m-1) c}\right)\right| \\
& \leq 8\left(1+x^{2}\right)\left(\mu_{n, 2}^{l, c}(x)+\frac{1}{\delta^{4}} \mu_{n, 6}^{l, c}(x)\right) \Omega\left(f^{\prime \prime}, \delta\right) \\
& \leq 8\left(1+x^{2}\right) \mu_{n, 2}^{l, c}(x)\left(1+\frac{1}{\delta^{4}} \frac{\mu_{n, 6}^{l, c}(x)}{\mu_{n, 2}^{l, c}(x)}\right) \Omega\left(f^{\prime \prime}, \delta\right) .
\end{aligned}
$$

Choosing $\delta=\left(\frac{\mu_{n, 6}^{l, c}(x)}{\mu_{n, 2}^{l, c}(x)}\right)^{1 / 4}$, we get the conclusion.

Following is the Grüss-Voronovskaya-type result.

Theorem 3.2. If $f, g \in C[0, \infty)$ and $f^{\prime \prime}, g^{\prime \prime} \in C_{2}^{*}[0, \infty)$, such that, $f g \in C[0, \infty)$ and $(f g)^{\prime \prime} \in C_{2}^{*}[0, \infty)$. Then for $x \in[0, \infty)$, we have

$$
\begin{aligned}
& n\left|R_{n, l, c}(f g, x)-R_{n, l, c}(f, x) R_{n, l, c}(g, x)-\mu_{n, 2}^{l, c}(x) f^{\prime}(x) g^{\prime}(x)\right| \\
\leq & 16\left(1+x^{2}\right) n \mu_{n, 2}^{l, c}(x)\left\{\Omega\left((f g)^{\prime \prime},\left(\frac{\mu_{n, 6}^{l, c}(x)}{\mu_{n, 2}^{l, c}(x)}\right)^{1 / 4}\right)\right.
\end{aligned}
$$




$$
\begin{aligned}
& +\|f\|_{2}\left(1+x^{2}\right) \Omega\left(g^{\prime \prime},\left(\frac{\mu_{n, 6}^{l, c}(x)}{\mu_{n, 2}^{l, c}(x)}\right)^{1 / 4}\right) \\
& \left.+\|g\|_{2}\left(1+x^{2}\right) \Omega\left(f^{\prime \prime},\left(\frac{\mu_{n, 6}^{l, c}(x)}{\mu_{n, 2}^{l, c}(x)}\right)^{1 / 4}\right)\right\}+n S_{n}(f) S_{n}(g),
\end{aligned}
$$

where $S_{n}(f)=\left\|f^{\prime \prime}\right\|_{2} \frac{\left(1+x^{2}\right)}{2}\left(2 \mu_{n, 2}^{l, c}(x)+\frac{2 x}{1+x^{2}} \mu_{n, 3}^{l, c}(x)+\frac{1}{1+x^{2}} \mu_{n, 4}^{l, c}(x)\right)$.

Proof. Applying Taylor expansion of $f$, using the fact that $R_{n, l, c}\left(e_{i}, x\right)=e_{i}, e_{i}(y)=y^{i}$ for $i=0,1$, and $(f g)^{\prime \prime}(x)=f^{\prime \prime}(x) g(x)+2 f^{\prime}(x) g^{\prime}(x)+g^{\prime \prime}(x) f(x)$, we have

$$
\begin{aligned}
& R_{n, l, c}(f g, x)-R_{n, l, c}(f, x) R_{n, l, c}(g, x)-R_{n, l, c}\left((t-x)^{2}, x\right) f^{\prime}(x) g^{\prime}(x) \\
= & {\left[R_{n, l, c}(f g, x)-f(x) g(x)-\frac{(f g)^{\prime \prime}(x)}{2 !} R_{n, l, c}\left((t-x)^{2}, x\right)\right] } \\
& -f(x)\left[R_{n, l, c}(g, x)-g(x)-\frac{g^{\prime \prime}(x)}{2 !} R_{n, l, c}\left((t-x)^{2}, x\right)\right] \\
& -g(x)\left[R_{n, l, c}(f, x)-f(x)-\frac{f^{\prime \prime}(x)}{2 !} R_{n, l, c}\left((t-x)^{2}, x\right)\right] \\
& +\left(g(x) R_{n, l, c}(g, x)\right) \cdot\left(R_{n, l, c}(f, x)-f(x)\right) \\
:= & S_{1}+S_{2}+S_{3}+S_{4} .
\end{aligned}
$$

Next,

$$
\begin{aligned}
& \left|R_{n, l, c}(f g, x)-R_{n, l, c}(f, x) R_{n, l, c}(g, x)-R_{n, l, c}\left((t-x)^{2}, x\right) f^{\prime}(x) g^{\prime}(x)\right| \\
\leq & \left|S_{1}\right|+\left|S_{2}\right|+\left|S_{3}\right|+\left|S_{4}\right| .
\end{aligned}
$$

By Theorem 3.1, we have the following estimates

$$
\begin{aligned}
& \left|S_{1}\right| \leq 16\left(1+x^{2}\right) \Omega\left((f g)^{\prime \prime},\left(\frac{\mu_{n, 6}^{l, c}(x)}{\mu_{n, 2}^{l, c}(x)}\right)^{1 / 4}\right) \mu_{n, 2}^{l, c}(x), \\
& \left|S_{2}\right| \leq|f(x)| 16\left(1+x^{2}\right) \Omega\left(g^{\prime \prime},\left(\frac{\mu_{n, 6}^{l, c}(x)}{\mu_{n, 2}^{l, c}(x)}\right)^{1 / 4}\right) \mu_{n, 2}^{l, c}(x), \\
& \left|S_{3}\right| \leq|g(x)| 16\left(1+x^{2}\right) \Omega\left(f^{\prime \prime},\left(\frac{\mu_{n, 6}^{l, c}(x)}{\mu_{n, 2}^{l, c}(x)}\right)^{1 / 4}\right) \mu_{n, 2}^{l, c}(x) .
\end{aligned}
$$

Now, as $f^{\prime \prime} \in C_{2}^{*}[0, \infty)$,

$$
R_{n, l, c}(f, x)-f(x)=f^{\prime}(x) \mu_{n, 1}^{l, c}(x)+\frac{1}{2} R_{n, l, c}\left(f^{\prime \prime}(\xi)(t-x)^{2}, x\right) .
$$


So,

$$
\begin{aligned}
\left|R_{n, l, c}(f, x)-f(x)\right| & \leq \frac{1}{2} R_{n, l, c}\left(\left|f^{\prime \prime}(\xi)\right|(t-x)^{2}, x\right) \\
& \leq\left\|f^{\prime \prime}\right\|_{2} \frac{1}{2} R_{n, l, c}\left(\left(1+\xi^{2}\right)(t-x)^{2}, x\right)
\end{aligned}
$$

where $\xi$ is a number between $t$ and $x$. There are two possible cases now.

If $t<\xi<x$, then $1+\xi^{2} \leq 1+x^{2}$. So, we get

$$
\left|R_{n, l, c}(f, x)-f(x)\right| \leq\left\|f^{\prime \prime}\right\|_{2} \frac{\left(1+x^{2}\right)}{2} \mu_{n, 2}^{l, c}(x) .
$$

If $x<\xi<t$, then $1+\xi^{2} \leq 1+t^{2}$. So, we get

$$
\begin{aligned}
\left|R_{n, l, c}(f, x)-f(x)\right| & \leq\left\|f^{\prime \prime}\right\|_{2} \frac{1}{2} R_{n, l, c}\left(\left(1+t^{2}\right)(t-x)^{2}, x\right) \\
& =\left\|f^{\prime \prime}\right\|_{2} \frac{1}{2}\left(\left(1+x^{2}\right) \mu_{n, 2}^{l, c}(x)+2 x \mu_{n, 3}^{l, c}(x)+\mu_{n, 4}^{l, c}(x)\right) .
\end{aligned}
$$

Combining these two cases, we obtain

$$
\begin{aligned}
\left|R_{n, l, c}(f, x)-f(x)\right| & \leq\left\|f^{\prime \prime}\right\|_{2} \frac{\left(1+x^{2}\right)}{2}\left(2 \mu_{n, 2}^{l, c}(x)+\frac{2 x}{1+x^{2}} \mu_{n, 3}^{l, c}(x)+\frac{1}{1+x^{2}} \mu_{n, 4}^{l, c}(x)\right) \\
& :=S_{n}(f) .
\end{aligned}
$$

Similarly, we can obtain $\left|R_{n, l, c}(g, x)-g(x)\right| \leq S_{n}(g)$ and hence, we get the desired result.

\section{Difference of Operators}

We compute the magnitude of difference of the two operators having the same basis function, viz. the generalized Baskakov operators and the genuine Gupta-Srivastava operators in this section. Varied researchers have studied in this direction (cf. $[3,7]$ and references therin).

Consider

$$
M_{n, l, c}(f, x)=\sum_{k=0}^{\infty} p_{n+l c, k}(x, c) G_{n, k}(f)
$$

and

$$
R_{n, l, c}(f, x)=\sum_{k=0}^{\infty} p_{n+l c, k}(x, c) H_{n, k}(f),
$$

where $G_{n, k}(f)=f\left(\frac{k}{n}\right)$ and $H_{n, k}(f)=[n+(l+1) c] \int_{0}^{\infty} p_{n+(l+2) c, k-1}(t, c) f(t) d t$, $1 \leq k<\infty, H_{0}(f)=f(0)$.

Remark 4.1. By simple computation, we have $b^{G_{n, k}}:=G_{n, k}\left(e_{1}\right)=\frac{k}{n}$ and

$$
\mu_{2}^{G_{n, k}}:=G_{n, k}\left(e_{1}-b^{G_{n, k}} e_{0}\right)^{2}=0 \quad \text { and } \quad \mu_{4}^{G_{n, k}}:=G_{n, k}\left(e_{1}-b^{G_{n, k}} e_{0}\right)^{4}=0 .
$$


Now,

$$
\begin{aligned}
H_{n, k}\left(e_{r}\right) & =[n+(l+1) c] \int_{0}^{\infty} p_{n+(l+2) c, k-1}(t, c) t^{r} d t \\
& =[n+(l+1) c]\left(\begin{array}{c}
\frac{n}{c}+l+k \\
k-1
\end{array}\right) \int_{0}^{\infty} \frac{(c t)^{k-1}}{(1+c t)^{\frac{n}{c}+l+k+1}} t^{r} d t \\
& =\frac{[n+(l+1) c]}{c^{r}}\left(\begin{array}{c}
\frac{n}{c}+l+k \\
k-1
\end{array}\right) \int_{0}^{\infty} \frac{(c t)^{k+r-1}}{(1+c t)^{\frac{n}{c}+l+k+1}} d t \\
& =\frac{[n+(l+1) c]}{c^{r+1}}\left(\begin{array}{c}
\frac{n}{c}+l+k \\
k-1
\end{array}\right) B\left(k+r, \frac{n}{c}+l-r+1\right) \\
& =\frac{[n+(l+1) c]}{c^{r+1}}\left(\begin{array}{c}
\frac{n}{c}+l+k \\
k-1
\end{array}\right) \frac{\Gamma(k+r) \Gamma\left(\frac{n}{c}+l-r+1\right)}{\Gamma\left(\frac{n}{c}+l+k+1\right)} \\
& =\frac{[n+(l+1) c]}{c^{r+1}} \frac{(k+r-1) !}{(k-1) !} \frac{\Gamma\left(\frac{n}{c}+l-r+1\right)}{\Gamma\left(\frac{n}{c}+l+2\right)} .
\end{aligned}
$$

Remark 4.2. $b^{H_{n, k}}:=H_{n, k}\left(e_{1}\right)=\frac{k}{n+l c}$ and we have

$$
\begin{aligned}
\mu_{2}^{H_{n, k}} & :=H_{n, k}\left(e_{1}-b^{H_{n, k}} e_{0}\right)^{2}=H_{n, k}\left(e_{2}\right)+\left(\frac{k}{n+l c}\right)^{2}-2 H_{n, k}\left(e_{1}\right)\left(\frac{k}{n+l c}\right) \\
& =\frac{k[n+c(l+k)]}{(n+l c)^{2}[n+(l-1) c]}
\end{aligned}
$$

and

$$
\begin{aligned}
& \mu_{4}^{H_{n, k}}:=H_{n, k}\left(e_{1}-b^{H_{n, k}} e_{0}\right)^{4} \\
& =H_{n, k}\left(e_{4}\right)-4\left(\frac{k}{n+l c}\right) H_{n, k}\left(e_{3}\right)+6\left(\frac{k}{n+l c}\right)^{2} H_{n, k}\left(e_{2}\right) \\
& -4\left(\frac{k}{n+l c}\right)^{3} H_{n, k}\left(e_{1}\right)+\left(\frac{k}{n+l c}\right)^{4} \\
& k\left[\begin{array}{c}
-3 c^{3} k^{3}(l-1)(l-2)(l-3)+(k+1)(k+2)(k+3) l c^{3}- \\
(k+1)(k+2)(k-9) l c^{2} n+\left(18+17 k+k^{3}\right) l c n^{2}+3(2+k) n^{3} \\
+3 c^{2} k^{2}\left(2(k+1)(l-2)(l-3) l c+\left(12+k+2(k-5) l-(k-2) l^{2}\right) n\right) \\
+c k\left(-4(k+1)(k+2)(l-3) l c^{2}+2(k+1)(24-3 k-8 l+2 k l) l c n\right. \\
\left.+\left(6(k+4)-\left(k^{2}+8\right) l\right) n^{2}\right)
\end{array}\right] .
\end{aligned}
$$


As an application of Theorem A, we have the following quantitative estimate for the difference between the operators $M_{n, l, c}$ and $R_{n, l, c}$.

Theorem 4.1. Let $f^{(s)} \in C_{B}[0, \infty), s \in\{0,1,2\}$ and $x \in[0, \infty)$, then for $n, c \in \mathbb{N}$, we have

$$
\left|\left(M_{n, l, c}-R_{n, l, c}\right)(f, x)\right| \leq\left\|f^{\prime \prime}\right\| \alpha(x)+\omega\left(f^{\prime \prime}, \delta_{1}(x)\right)(1+\alpha(x))+2 \omega\left(f, \delta_{2}(x)\right),
$$

where

$$
\begin{aligned}
\alpha(x): & =\frac{1}{2} \sum_{k=0}^{\infty} p_{n+l c, k}(x, c)\left(\mu_{2}^{G_{n, k}}+\mu_{2}^{H_{n, k}}\right)=\frac{x(1+c x)[n+(l+1) c]}{2(n+l c)[n+(l-1) c]}, \\
\delta_{1}^{2}(x):= & \frac{1}{2} \sum_{k=0}^{\infty} p_{n+l c, k}(x, c)\left(\mu_{4}^{G_{n, k}}+\mu_{4}^{H_{n, k}}\right) \\
= & \frac{1}{2}\left(\begin{array}{l}
n+l c) x \\
\end{array}\right. \\
& \times\left[\begin{array}{c}
6(n+l c)^{3}-(8 c(l-3)-11 l c-3 n)(n+l c)^{2}(1+(n+(l+1) c) x) \\
+6(n+l c)\left(c^{2}(l-2)(l-3)+l c^{2}+c(-2(l-3) l c+n)\right) \\
(1+(n+(l+1) c) x(3+c(l+2) x+n x)) \\
-\left(3 c^{3}(l-1)(l-2)(l-3)+c(2 l c-n)(2(l-3) l c-l n)\right. \\
\left.-l c\left(l c^{2}-l c n+n^{2}\right)-3 c^{2}(n+(l-2)(2(l-3) l c-l n))\right) \\
(1+(n+(l+1) c) x(7+(c(l+2)+n) x(6+c(l+3) x+n x)))
\end{array}\right]
\end{aligned}
$$

and

$$
\delta_{2}^{2}(x):=\sum_{k=0}^{\infty} p_{n+l c, k}(x, c)\left(b^{G_{n, k}}-b^{H_{n, k}}\right)^{2}=\frac{l c x}{(n+l c)}\left(1+\frac{l c}{n}\right) .
$$

Proof. The proof immediately follows using Remark 2.1, 4.1 and 4.2. We omit the details.

Acknowledgements. The authors are thankful to the reviewers for valuable suggestions.

\section{REFERENCES}

[1] T. Acar, L. N. Mishra and V. N. Mishra, Simultaneous approximation for generalized SrivastavaGupta operators, J. Funct. Spaces (2015), Article ID 936308, 11 pages.

[2] A.-M. Acu and C. V. Muraru, Certain approximation properties of Srivastava-Gupta operators, J. Math. Inequal. 12(2) (2018), $583-595$.

[3] A. Aral, D. Inoan and I. Rasa, On differences of linear positive operators, Anal. Math. Phys. (2018), DOI 10.1007/s1332.

[4] N. Deo, Faster rate of convergence on Srivastava-Gupta operators, Appl. Math. Comput. 218(21) (2012), 10486-10491.

[5] A.-J. López-Moreno, J.-Manuel and L.-Palacios, Localization results for generalized Baskakov/Mastroianni and composite operators, J. Math. Anal. Appl. 380(2) (2011), 425-439. 
[6] N. K. Govil, V. Gupta and D. Soybaş, Certain new classes of Durrmeyer type operators, Appl. Math. Comput. 225 (2013), 195-203.

[7] V. Gupta, Difference of operators of Lupaş type, Constructive Mathematical Analysis 1(1) (2018), 9-14.

[8] V. Gupta, Some examples of genuine approximation operators, Gen. Math. 26(1-2) (2018), 3-9.

[9] V. Gupta, D. Soybaş and G. Tachev, Improved approximation on Durrmeyer-type operators, Bol. Soc. Mat. Mex. (3) (2018), 1-11.

[10] V. Gupta and H. M. Srivastava, A general family of the Srivastava-Gupta operators preserving linear functions, Eur. J. Pure Appl. Math. 11(3) (2018), 575-579.

[11] N. Ispir, On modified Baskakov operators on weighted spaces, Turk. J. Math. 25 (2001), 355-365.

[12] N. Ispir and I. Yuksel, On the Bézier variant of Srivastava-Gupta operators, Appl. Math. E-Notes 5 (2005), 129-137.

[13] N. Malik, Some approximation properties for generalized Srivastava-Gupta operators, Appl. Math. Comput. 269 (2015), 747-758.

[14] N. Malik, S. Araci and M. S. Beniwal, Approximation of Durrmeyer type operators depending on certain parameters, Abstr. Appl. Anal. (2017), Article ID 5316150, 9 pages.

[15] G. Mastroianni, Su un operatore lineare e positivo, Rend. Accad. Sci. Fis. Mat. Napoli (4) 46 (1979), 161-176.

[16] R. Pratap and N. Deo, Approximation by genuine Gupta-Srivastava operators, Rev. R. Acad. Cienc. Exactas FIs. Nat. Ser. A Math. RACSAM (2019), DOI 10.1007/s13398-019-00633-4.

[17] D. Soybaş, Approximation with modified Phillips operators, J. Nonlinear Sci. Appl. 10(11) (2017), 5803-5812.

[18] H. M. Srivastava and V. Gupta, A certain family of summation-integral type operators, Math. Comput. Modelling 37 (2003), 1307-1315.

[19] D. K. Verma and P. N. Agrawal, Convergence in simultaneous approximation for SrivastavaGupta operators, Math. Sci. 6 (2012), Article ID 22.

[20] R. Yadav, Approximation by modified Srivastava-Gupta operators, Appl. Math. Comput. 226 (2014), 61-66.

${ }^{1}$ Department of Mathematics Education, Faculty of Education,

ERCIYES UNIVERSITY,

KAYSERI 38039, TURKEY

Email address: danyal@erciyes.edu.tr

${ }^{2}$ Statistics and Mathematics Unit,

Indian Statistical Institute,

BANGALORE 560059, INDIA

Email address: neha.malik_nm@yahoo.com 